\title{
Sturm-Liouville difference equations having Bessel and hydrogen atom potential type
}

https://doi.org/10.1515/phys-2018-0100

Received January 27, 2018; accepted July 31, 2018

\begin{abstract}
In this work, we bring a different approach for Sturm-Liouville problems having Bessel and hydrogen atom type and we provide a basis for direct and inverse problems. From this point of view, we find representations of solutions and asymptotic expansions for eigenfunctions. Furthermore, some numerical estimations are given to illustrate the necessity of the Sturm-Liouville difference equations with the potential function for the convenience to the spectral theory. The behavior of eigenfunctions for the Sturm-Liouville problem having Bessel and hydrogen atom potential type is analyzed and compared to each other. And then, comparisons are showed by tables and figures.
\end{abstract}

Keywords: Sturm-Liouville; Difference equation; Bessel potential; Hydrogen atom potential; Schrödinger equation

PACS: 02.30.Tb; 02.90.+p; 02.30.Zz

\section{Introduction}

The Sturm-Liouville problem is a one-particle Schrödinger equation, and it has an important place in mathematical physics. The potential function $q(t)$ determines the type of equation, like Bessel and hydrogen atom equations. In this study, we consider Sturm-Liouville problems with two different potentials and investigate the behaviors of its eigenfunctions. The Liouville normal form of Bessel equations, also called Sturm-Liouville equation having Bessel poten-

\footnotetext{
^Corresponding Author: Erdal Bas: Firat University, Science Faculty, Department of Mathematics, 23119 Elazig, Turkey,

E-mail: erdalmat@yahoo.com

Ramazan Ozarslan: Firat University, Science Faculty, Department of Mathematics, 23119 Elazig, Turkey,

E-mail: ozarslanramazan@gmail.com

Dumitru Baleanu: Department of Mathematics and Computer Sciences, Faculty of Arts and Sciences, Cankaya University, Ankara, Turkey, Institute of Space Sciences, Magurele-Bucharest, Romania, E-mail: dumitru@cankaya.edu.tr
}

tial type, is studied in [1]-[9] , and it is defined as follows,

$$
y^{\prime \prime}+\left(\lambda-\frac{p^{2}-\frac{1}{4}}{x^{2}}-q(x)\right) y=0
$$

Bessel functions are obtained by series solutions while $q(x)=0$ in the equation above, accordingly, the equation above is more general than classical Bessel equation. As $q(x)$ changes, the structure of the equation will change and hence, a different investigation will require. Lately, the Bessel difference equation has been studied by [10], and obtained discrete Bessel functions, which are the discrete analogue of Bessel functions. The radial Schrödinger equation, also called hydrogen atom equation, is studied by $[3,7,8,11,12]$, and it is defined as follows,

$$
y^{\prime \prime}+\left(\lambda-\frac{\ell(\ell+1)}{x^{2}}+\frac{2}{x}-q(x)\right) y=0 .
$$

Physical interpretation of hydrogen atom and Bessel equations:

Bessel equations seem to have a lot of application areas in physics and mathematics. For example, the solution of two-dimensional wave equation, the heat equation and the Dirichlet problem in a cylinder are obtained with the help of Bessel functions [4, 13, 14]. Also, vibrational, gravitational and electromagnetic potential problems with cylindrical symmetry, diffraction problems (astronomy) resolving power of optical instruments, heavy chain, certain subjects in chemistry and biochemistry are expressed by the means of Bessel equations [5].

The radial Schrödinger equation enables to calculate the development of quantum systems with time, also it can give analytical solutions for the non-relativistic hydrogen atom.

$\frac{d^{2} R}{d r^{2}}+\frac{2}{r} \frac{d R}{d r}-\frac{\ell(\ell+1)}{r^{2}} R+\left(E+\frac{2}{r}\right) R=0 \quad(0<r<\infty)$,

where $R$ is the distance of the mass center to the origin, $\ell$ is the orbital quantum number and a positive integer, $E$ is energy constant and $r$ is the distance between the nucleus with the electron.

The hydrogen atom is a system consists of a twoparticle system, and it forms of one electron and one proton. Internal motion of two particles around the center of 
mass corresponds to the movement of a single particle by a reduced mass. If $R=\frac{y}{x}$ and $E=\lambda$ is taken in the equation above, it forms of

$$
y^{\prime \prime}+\left(\lambda-\frac{\ell(\ell+1)}{x^{2}}+\frac{2}{x}-q(x)\right) y=0 .
$$

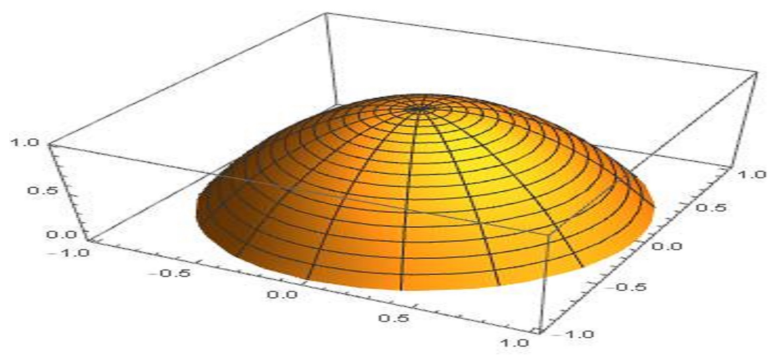

Figure 1: Bessel functions are the radial part of modes of vibrations of a circular drum[9]

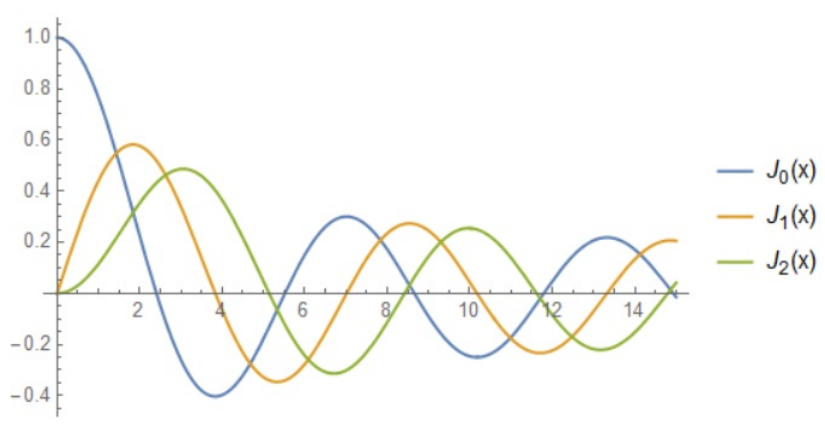

Figure 2: The Bessel functions of the first kind

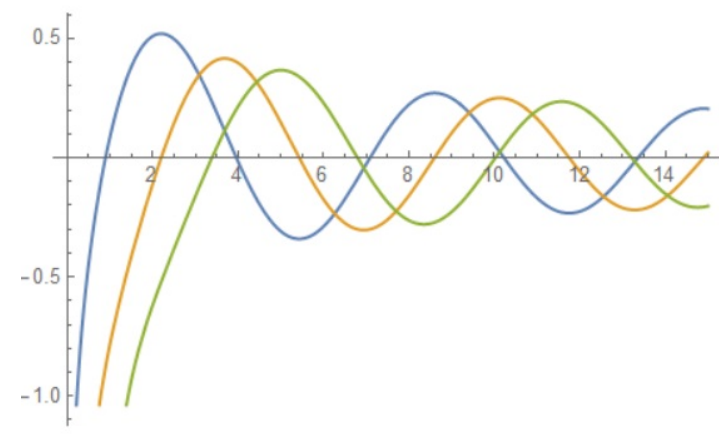

$-Y_{0}(x)$

$-Y_{1}(x)$

$-Y_{2}(x)$

Figure 3: The Bessel functions of the second kind
Difference equations have always been an interesting subject due to the fact that the discrete analogue of differential equations. The theory of linear ordinary difference equations was improved by [15]-[19]. Recently, spectral analysis of difference equations has attracted great attention. Especially, many scientists study on Sturm-Liouville difference equations, see [16]-[18],[20]-[25].

The zeros of Bessel and hydrogen atom problems cannot be calculated directly because of having closed form solutions, and this type of solution is called the representation of solution. Accordingly, solution function $y(x)$, also called eigenfunction, can be found only by asymptotic estimations. Based on this, the number of eigenvalues $\lambda$ can be found only by asymptotic estimations. Thereafter, the other spectral data, like norming constants, normalized eigenfunctions and spectral function, are found by asymptotic estimations. If we close attention, we have no knowledge about potential function $q(x)$ except for it is a continuous function. This type of problem is named "direct problem". Viceversa, while there is knowledge about spectral data, one is made estimations about potential function $q(x)$ and this type of problem is named "inverse problem". Additionally, Bessel functions are obtained by series solutions while $q(x)=0$ in the equation above, accordingly the equation above is more general than classical Bessel equation. As $q(x)$ changes, structure of the equation will change and hence, a different investigation will require. Our main aim is to apply the spectral theory of these type of differential equations to the discrete case.

In this paper, we are concerned with the discrete analogue of Sturm-Liouville equations having hydrogen atom and Bessel potentials, and we provide a basis for direct and inverse problems. From this point of view, we obtain representation of solutions, asymptotic estimations of eigenfunctions and some numerical estimations about behaviors of eigenfunctions and eigenvalues. The numerical results for the eigenfunctions corresponding to the certain significant eigenvalues for Sturm-Liouville problem having Bessel and hydrogen atom potential type are shown and compared to the each other. The integral representation and asymptotic formulae for eigenfunctions of Sturm-Liouville differential problem are found in [3]. Similarly, the sum representation and asymptotic formulae for eigenfunctions of Sturm-Liouville difference problem are acquired in [26]-[28]. Also, numerical computations of Sturm-Liouville problem are considered in [21], [29]. 
Now, let's introduce Liouville normal form of Bessel's difference equations

$\Delta^{2} u(n-1)+\left(\lambda+q(n)-\frac{p^{2}-\frac{1}{4}}{n^{2}}\right) u(n)=0, n=1, \ldots, b$,

where $p \in \mathbb{R}, u(n), q(n) \in l^{2}[0, b], b$ is a finite integer, $\Delta$ is the forward difference operator, $\Delta x(n)=x(n+1)-x(n)$, assume that $\lambda$ is the positive spectral parameter, $q(n)-$ $\frac{p^{2}-\frac{1}{4}}{n^{2}}$ are called potential function, $n$ is a finite integer. Then, let's introduce hydrogen atom difference equation

$$
\begin{aligned}
\Delta^{2} v(n-1)+\left(\lambda-q(n)+\frac{2}{n}-\frac{\ell(\ell+1)}{n^{2}}\right) & v(n)=0, \\
n & =1, \ldots, b,
\end{aligned}
$$

where $\ell$ is the orbital quantum number and a positive integer, $v(n) \in \ell^{2}[0, b], q(n), b, \lambda$ and $n$ is as defined above, $-q(n)+\frac{2}{n}-\frac{\ell(\ell+1)}{n^{2}}$ are called potential function.

Our object is to give discrete analogue results to the studies mentioned before [2], [3], [26]-[28], [30].

\section{Preliminaries}

Definition 1. [25] The matrix of Casoratian is defined by

$$
\begin{aligned}
& w(n)= \\
& \left(\begin{array}{ccc}
u_{1}(n) & u_{2}(n) \ldots & u_{r}(n) \\
u_{1}(n+1) & u_{2}(n+1) \ldots & u_{r}(n+1) \\
\vdots & \vdots & \vdots \\
u_{1}(n+r-1) & u_{2}(n+r-1) \ldots & u_{r}(n+r-1)
\end{array}\right)
\end{aligned}
$$

where $u_{1}(n), u_{2}(n), \ldots, u_{r}(n)$ are known functions. The determinant

$$
W(n)=\operatorname{det} w(n)
$$

is called Casoratian.

Theorem 1. [16] (Wronskian-Type Identity) Assume y and $y$ are linearly independent solutions of Sturm-Liouville difference equation, mentioned in [26]. Then, for $a \leq n \leq b$

$$
\begin{aligned}
W[y, z](n) & =[y(n) \Delta z(n-1)-y(n) \Delta z(n-1)] \\
& =-[y(n) z(n-1)-y(n-1) z(n)]
\end{aligned}
$$

is a constant (Particularly equal to $W[r, u](a)$ ).

Definition 2. Let's define Sturm-Liouville problem having Bessel potential type (1) as following, $n \in[1, b]$

$$
L_{1} u(n)=\Delta^{2} u(n-1)+\left(q(n)-\frac{p^{2}-\frac{1}{4}}{n^{2}}\right) u(n)=-\lambda u(n),
$$

with initial conditions

$$
u(1)=-h, u(2)=1,
$$

where $L_{1}$ is a self-adjoint difference operator, $h=\cot \alpha, \alpha \in$ $\mathbb{R}$.

Definition 3. Let's define Sturm-Liouville problem having hydrogen atom potential (2) as following, $n \in[1, b]$,

$$
\begin{aligned}
L_{2} v(n) & =\Delta^{2} v(n-1)+\left(-q(n)+\frac{2}{n}-\frac{\ell(\ell+1)}{n^{2}}\right) v(n) \\
& =-\lambda v(n),
\end{aligned}
$$

with initial conditions

$$
v(1)=-h, v(2)=1,
$$

where $L_{2}$ is a self-adjoint difference operator, $h=\cot \alpha, \alpha \in$ $\mathbb{R}$.

Theorem 2. [25] (Summation by parts) Assume $m<n$, then

$$
\sum_{k=m}^{n-1} u(k) \Delta r(k)=[u(k) r(k)]_{m}^{n}-\sum_{k=m}^{n-1} \Delta u(k) r(k+1) .
$$

Theorem 3. [25] Assume $z_{n}$ is an indefinite sum of $u_{n}$, then

$$
\sum_{k=m}^{n-1} z(k)=u(n)-u(m) .
$$

\section{Main results}

In this paper, we are concerned with the discrete analogue of Sturm-Liouville problem with Bessel and hydrogen atom potentials (6)-(7), (4) - (5), and we provide a basis for direct and inverse problems. From this point of view, we obtain the representation of solutions, asymptotic estimations of eigenfunction and some numerical estimations about behaviors of eigenfunctions and eigenvalues. The numerical results for eigenfunctions corresponding to the certain significant eigenvalues for Sturm-Liouville problem having Bessel and hydrogen atom potential type are shown and compared to the each other. Our main aim is to apply the spectral theory of these type of differential equations to the discrete case.

In $\ell^{2}(1, b)$, the Hilbert space of sequences of complex numbers $u(1), \ldots, u(b)$ with the inner product,

$$
<u(n), r(n)>=\sum_{n=1}^{b} u(n) r(n),
$$


for every $u \in D_{L}$, let's define as follows

$$
\begin{aligned}
D_{L}= & \left\{u(n), v(n) \in \ell^{2}(1, b): L_{1} u(n),\right. \\
& L_{2} v(n) \in \ell^{2}(1, b), u(1)=v(1)=-h, \\
& u(2)=v(2)=1 .\}
\end{aligned}
$$

In this section, we show the sum representations of solutions of Sturm-Liouville problem having Bessel and hydrogen atom potential by the variation of parameters method and prove that the solutions obtained hold for the problems.

Theorem 4. Sturm-Liouville problem having Bessel potential type is defined as follows

$$
\begin{gathered}
\Delta^{2} \varphi(n-1)+\left(q(n)-\frac{p^{2}-\frac{1}{4}}{n^{2}}\right) \varphi(n)=-\lambda \varphi(n), \\
\varphi(1)=-h, \varphi(2)=1,
\end{gathered}
$$

then the problem (10) - (11) has a unique solution for $\varphi(n)$ as follows,

$\varphi(n, \lambda)=\left(\frac{-h \sin 2 \theta-\sin \theta\left[1+h\left(q(1)+p^{2}-\frac{1}{4}\right)\right]}{\sin \theta}\right) \cos n \theta$

$$
\begin{gathered}
+\left(\frac{\cos \theta\left[1+h\left(q(1)+p^{2}-\frac{1}{4}\right)\right]+h \cos 2 \theta}{\sin \theta}\right) \sin n \theta \\
+\frac{1}{\sin \theta} \sum_{i=1}^{n-1}\left(-q(i)+\frac{p^{2}-\frac{1}{4}}{i^{2}}\right) \varphi(i) \sin (n-i) \theta,
\end{gathered}
$$

where, $\sum_{i=a}^{b} .=0$ if $a>b$.

Proof. We restrict the eigenvalues with $0<\lambda<4$ to be able to obtain more advantageous results for the spectral theory. Let $u_{1}(n)$ and $u_{2}(n)$ be linearly idependent solutions for homogeneous part of (10), so by characteristic equation, we can find characteristic roots, and since $|\lambda-2|<2$, we can take

$$
\lambda=2-2 \cos \theta,
$$

from here, the solution of the homogeneous part of (10) is

$$
\varphi_{h}(n)=c_{1} \cos n \theta+c_{2} \sin n \theta .
$$

By means of variation of parameters method for difference equations [2, 17], we get

$$
\varphi_{p}(n)=c_{1}(n) \cos n \theta+c_{2}(n) \sin n \theta,
$$

if we insert the equation (12) in the equation (10) and make necessary operations, then we can find the parameters as,

$$
c_{1}(n)=\sum_{i=1}^{n} \frac{\left[-q(i)+\frac{p^{2}-\frac{1}{4}}{i^{2}}\right] \varphi(i) \varphi_{2}(i)}{W},
$$

$$
c_{2}(n)=-\sum_{i=1}^{n} \frac{\left[-q(i)+\frac{p^{2}-\frac{1}{4}}{i^{2}}\right] \varphi(i) \varphi_{1}(i)}{W},
$$

where $W=\sin \theta$ by Theorem 1 .

Eventually, we get the general solution

$$
\begin{aligned}
& \varphi(n, \lambda)=c_{1} \cos n \theta+c_{2} \sin n \theta \\
& +\frac{1}{\sin \theta} \sum_{i=1}^{n}\left[-q(i)+\frac{p^{2}-\frac{1}{4}}{i^{2}}\right] \varphi(i) \sin (n-i) \theta,
\end{aligned}
$$

by the initial conditions, we get the sum representation of solution, $c_{1} \neq 0, c_{2} \neq 0$,

$$
\begin{gathered}
\varphi(n, \lambda)=\left(\frac{-h \sin 2 \theta-\sin \theta\left[1+h\left(q(1)+p^{2}-\frac{1}{4}\right)\right]}{\sin \theta}\right) \cos n \theta \\
+\left(\frac{\cos \theta\left[1+h\left(q(1)+p^{2}-\frac{1}{4}\right)\right]+h \cos 2 \theta}{\sin \theta}\right) \sin n \theta+ \\
+\frac{1}{\sin \theta} \sum_{i=1}^{n-1}\left(-q(i)+\frac{p^{2}-\frac{1}{4}}{i^{2}}\right) \varphi(i) \sin (n-i) \theta .
\end{gathered}
$$

We can show that the solution holds for the problem (10)(11) by inserting in (10) by virtue of Theorem 2 and Theorem 3.

The proof completes.

Theorem 5. Sturm-Liouville problem having hydrogen atom potential type is defined as follows

$$
\Delta^{2} \psi(n-1)+\left(-q(n)+\frac{2}{n}-\frac{\ell(\ell+1)}{n^{2}}\right) \psi(n)=-\lambda \psi(n),
$$

$$
\psi(1)=-h, \psi(2)=1,
$$

then the problem (14) - (15) has a unique solution for $\psi(n)$ as follows,

$$
\begin{aligned}
& \psi(n, \lambda)= \\
& \left(\frac{-h \sin 2 \theta-\sin \theta[1+h(q(1)+2+\ell(\ell+1))]}{\sin \theta}\right) \cos n \theta \\
& +\left(\frac{\cos \theta[1+h(q(1)+2+\ell(\ell+1))]+h \cos 2 \theta}{\sin \theta}\right) \sin n \theta \\
& +\frac{1}{\sin \theta} \sum_{i=1}^{n-1}\left(q(i)+\frac{2}{i}+\frac{\ell(\ell+1)}{i^{2}}\right) \psi(i) \sin (n-i) \theta .
\end{aligned}
$$

where, $\sum_{i=a}^{b} .=0$ if $a>b$.

Proof. Similar arguments used in the proof of Theorem 5 are applied to easily obtain the conclusion. 


\section{Asymptotic formulas for Bessel and hydrogen atom difference equations}

In this section, we give the asymptotic formulas for the eigenfunctions of Bessel and hydrogen atom potential, respectively.

Theorem 6. Sturm-Liouville problem having Bessel potential (10) - (11) has the estimate

$$
\varphi(n)=O\left(e^{|\tau| n}\right),
$$

and more precisely

$$
\begin{aligned}
\varphi(n)= & \left(\frac{-h \sin 2 \theta-\sin \theta\left[1+h\left(q(1)+p^{2}-\frac{1}{4}\right)\right]}{\sin \theta}\right) \cos n \theta \\
& +O\left(\frac{e^{|\tau| n}}{\sin \frac{\theta}{2} \sin \theta}\right),
\end{aligned}
$$

where $n \in \mathbb{Z}^{+},\{\varphi(n)\}$ is a complex sequence, $\sum_{i=0}^{\infty} i|q(i)|<$ $\infty$

Proof. Let's insert $\varphi(n)=f(n) e^{|\tau| n}$ in (10). Hence, we get

$$
\begin{gathered}
f(n)=\left(\frac{-h \sin 2 \theta-\sin \theta\left[1+h\left(q(1)+p^{2}-\frac{1}{4}\right)\right]}{\sin \theta}\right) e^{-|\tau| n} \\
+\left(\frac{\cos \theta\left[1+h\left(q(1)+p^{2}-\frac{1}{4}\right)\right]+h \cos 2 \theta}{\sin \theta}\right) e^{-|\tau| n} \\
+\frac{1}{\sin \theta} \sum_{i=1}^{n-1}\left(-q(i)+\frac{p^{2}-\frac{1}{4}}{i^{2}}\right) f(i) e^{|\tau|(n-i)} \sin (n-i) \theta .
\end{gathered}
$$

Let $\xi=\max _{1 \leq n \leq \infty}|f(n)|$, then if we insert the last equation in that

$$
\begin{gathered}
\xi \leq 2\left(|h|+1+|h|\left(|q(1)|+p^{2}+\frac{1}{4}\right)\right) \\
+\frac{\xi}{|\sin \theta|} \sum_{i=1}^{\infty}\left(|q(i)|+\frac{p^{2}+\frac{1}{4}}{i^{2}}\right),
\end{gathered}
$$

and thus,

$$
\xi \leq \frac{2\left(|h|+1+|h|\left(|q(1)|+p^{2}+\frac{1}{4}\right)\right)}{1-\frac{1}{|\sin \theta|} \sum_{i=1}^{\infty}|| q(i)\left|+\frac{p^{2}+\frac{1}{4}}{i^{2}}\right|},
$$

the proof completes under the assumption that nominator of the last inequality is large enough and

$$
|\sin \theta|>\sum_{i=1}^{\infty}|| q(i)\left|+\frac{p^{2}+\frac{1}{4}}{i^{2}}\right| .
$$

The proof is complete under the assumption.
Theorem 7. Sturm-Liouville problem having hydrogen atom potential (14) - (15) has the estimate

$$
\psi(n)=O\left(e^{|\tau| n}\right),
$$

and more precisely

$$
\begin{array}{r}
\psi(n)=\left(\frac{-h \sin 2 \theta-\sin \theta[1+h(q(1)+2+\ell(\ell+1))]}{\sin \theta}\right) \cos n \theta \\
+O\left(\frac{e^{|\tau| n}}{\sin \frac{\theta}{2} \sin \theta}\right),
\end{array}
$$

where $n \in \mathbb{Z}^{+},\{\psi(n)\}$ is a complex sequence, $\sum_{i=0}^{\infty} i|q(i)|<$ $\infty$.

Proof. Similar arguments used in the proof of Theorem 6 are applied to easily obtain the conclusion.

\section{Applications}

Assuming that $h=p=1, l=2, b=24$ in all of the applications.

Application 1. Taking $q_{1}(n)=\frac{1}{\sqrt{n}}$ in the problems (10) (11) and (14) - (15), then we have the eigenfunctions as in Figure 4, Figure 5, Figure 6, Table 1, Table 2, Table 3 Application 2. Taking $h=p=l=1, b=24$ and $q_{2}(n)=$ 1 in the problem (10) - (11) and (14) - (15), we have the eigenfunctions as in Table4, Table5.

Application 3. Taking $h=p=1, l=2, b=24$ and $q_{3}(n)=$ $\frac{1}{n}$ in the problem (10) - (11) and (14) - (15), we have the eigenfunctions as in Fig8-Fig17.

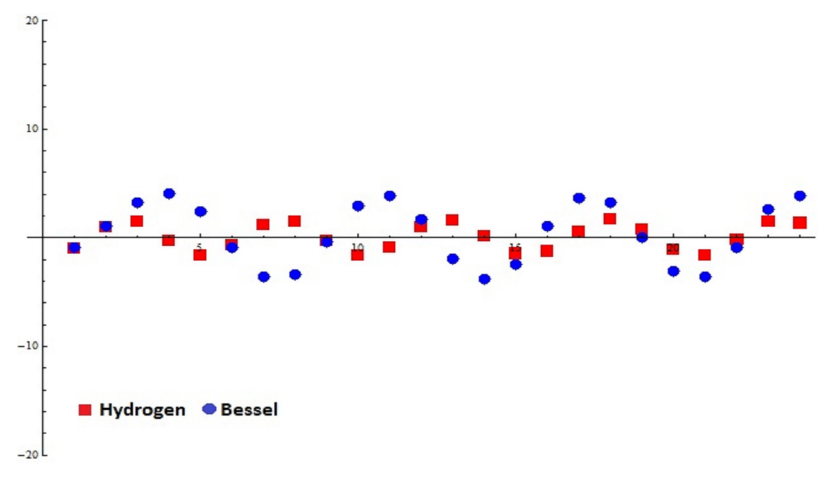

Figure 4: Comparison of datas in Table 1 


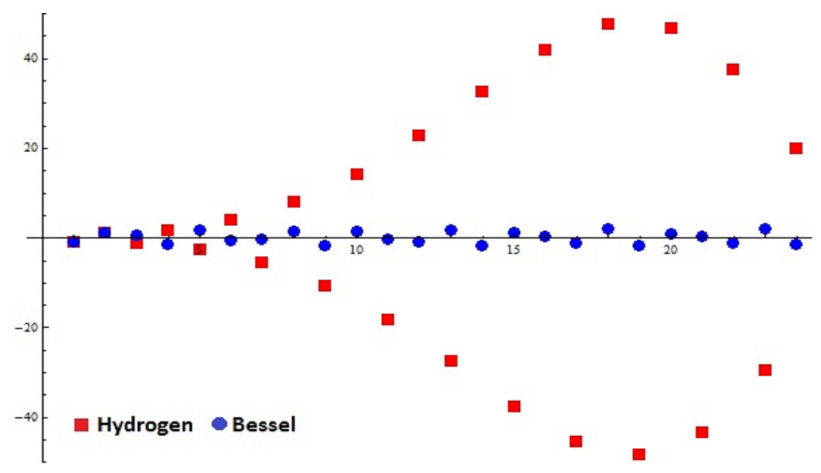

Table 1

Figure 5: Comparison of datas in Table 3

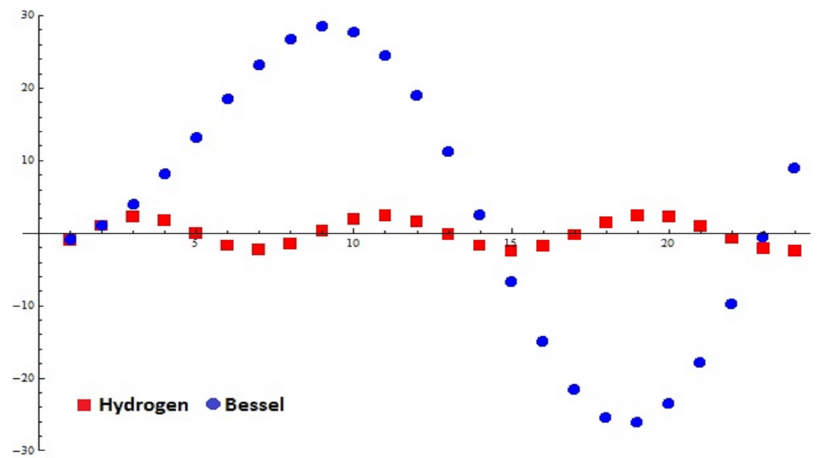

Figure 6: Comparison of datas in Table 2

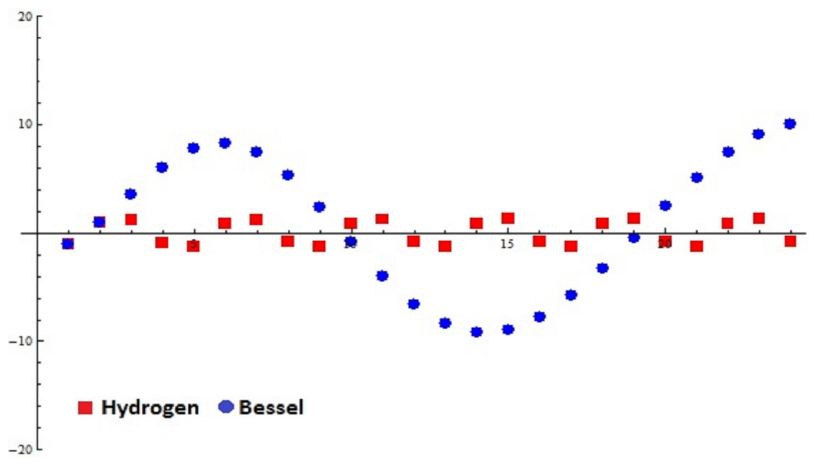

Figure 7: Comparison of datas in Table 4

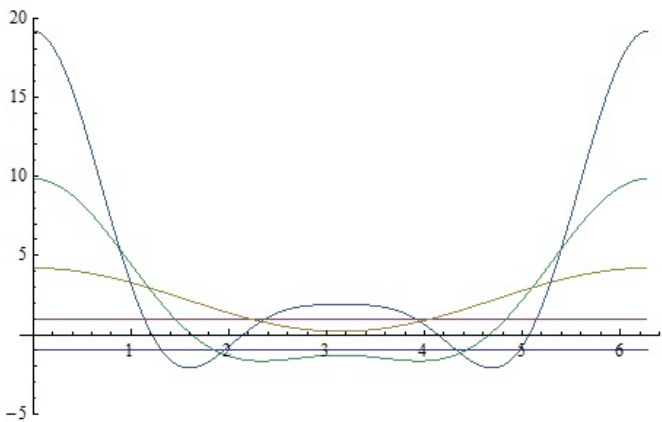

Figure 8: $\psi(n), n=1,2, \ldots, 5$

Table 2

\begin{tabular}{ccc}
\multicolumn{3}{c}{ Eigenfunctions correspond to $\lambda_{2}=2-\sqrt{3}$} \\
\hline$n$ & Bessel: $\varphi(n)$ & Hydrogen: $\psi(n)$ \\
\hline 1 & -1 & -1 \\
2 & 1 & 3.93916 \\
3 & 2.21244 & 8.09709 \\
4 & 1.73908 & 13.1218 \\
5 & 0.0117111 & 18.3994 \\
6 & -1.72368 & 11.2468 \\
13 & -0.145179 & 2.4093 \\
14 & -1.75401 & -6.70029 \\
15 & -2.43079 & -15.0299 \\
16 & -1.83671 & -17.9065 \\
21 & 0.930821 & -9.8949 \\
22 & -0.785314 & -0.564677 \\
23 & -2.12481 & 8.84181 \\
24 & -2.45493 &
\end{tabular}

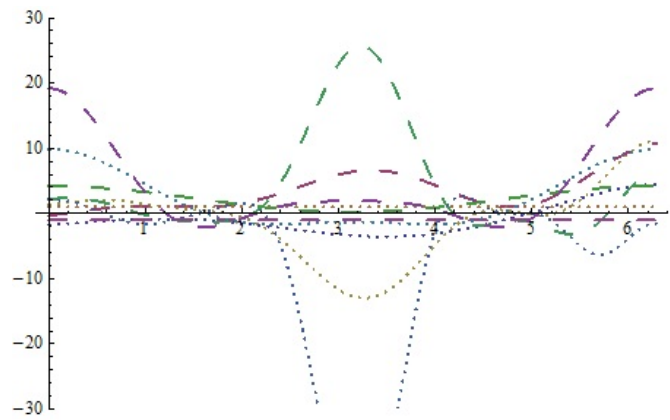

Figure 9: Comparison of $\varphi(n), n=1,2, \ldots, 5$ 
Table 3

\begin{tabular}{ccc}
\hline \multicolumn{3}{c}{ Eigenfunctions correspond to $\lambda_{3}=2+\sqrt{3}$} \\
\hline$n$ & Bessel: $\varphi(n)$ & Hydrogen: $\psi(n)$ \\
\hline 1 & -1 & -1 \\
2 & 1 & 1 \\
3 & -1.25166 & 0.475056 \\
4 & 1.78627 & -1.54855 \\
5 & -2.65167 & 1.6264 \\
6 & 3.91286 & -0.801338 \\
13 & -27.6413 & 1.69851 \\
14 & 32.6601 & -1.76153 \\
15 & -37.5314 & 1.07949 \\
16 & 41.9117 & 0.055379 \\
21 & -43.325 & 0.271728 \\
22 & 37.5582 & -1.32168 \\
23 & -29.6769 & 1.83948 \\
24 & 19.9896 & -1.61992 \\
\hline
\end{tabular}

Table 4

\begin{tabular}{ccc}
\hline \multicolumn{3}{c}{ Eigenfunctions correspond to $\lambda_{1}=1$} \\
\hline$n$ & Bessel: $\varphi(n)$ & Hydrogen: $\psi(n)$ \\
\hline 1 & -1 & -1 \\
2 & 1 & 1 \\
3 & 1.1875 & 3.5 \\
4 & -0.901042 & 6 \\
5 & -1.22974 & 7.75 \\
6 & 0.86415 & 8.26 \\
13 & -1.26819 & -8.39808 \\
14 & 0.819945 & -9.19431 \\
15 & 1.27133 & -8.95851 \\
16 & -0.815707 & -7.76715 \\
21 & -1.27712 & 5.13985 \\
22 & 0.807579 & 7.3874 \\
23 & 1.27837 & 9.05495 \\
24 & -0.805767 & 10.0378 \\
\hline
\end{tabular}

Table 5

\begin{tabular}{ccc}
\hline \multicolumn{3}{c}{ Eigenfunctions correspond to $\lambda_{5}=2-\sqrt{3}$} \\
\hline$n$ & Bessel: $\varphi(n)$ & Hydrogen: $\psi(n)$ \\
\hline 1 & -1 & -1 \\
2 & 1 & 1 \\
3 & 2.41955 & 3.73205 \\
4 & 2.5859 & 6.70812 \\
5 & 1.53409 & 8.72527 \\
6 & -0.189564 & 8.7535 \\
13 & 2.73757 & -4.85591 \\
14 & 2.81137 & -0.212425 \\
15 & 1.94182 & 4.49665 \\
16 & 0.428975 & 7.82098 \\
21 & -1.20795 & -6.02999 \\
22 & 0.42675 & -8.51653 \\
23 & 1.92836 & -8.43953 \\
24 & 2.83217 & -5.82995 \\
\hline
\end{tabular}

- $\lambda=1 \quad \lambda=2-\sqrt{3} \quad \lambda=2-\sqrt{2}$

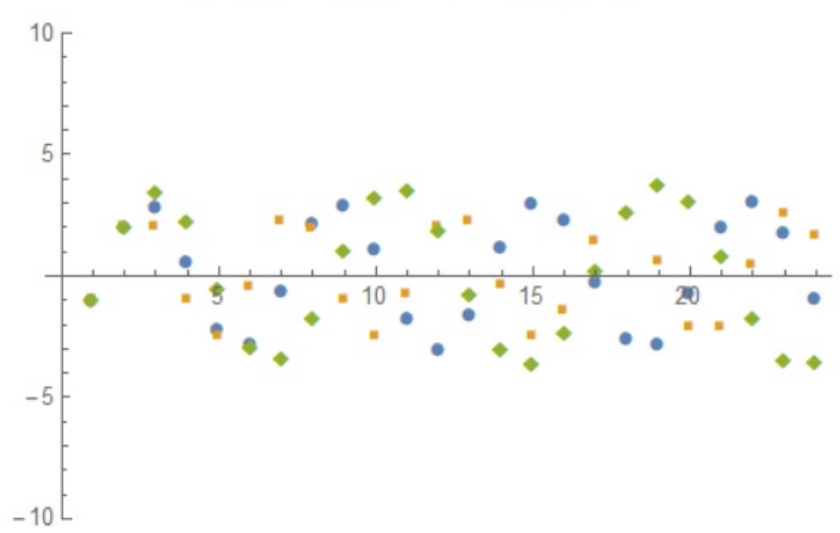

Figure 10: Comparison of $\varphi(n)$ and $\psi(n), \mathrm{n}=1,2, \ldots, 5$

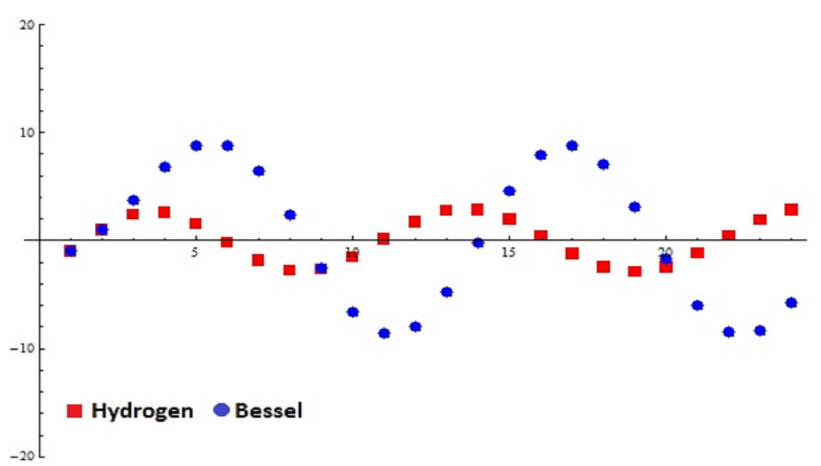

Figure 11: Comparison of datas in Table 5 
- $p=-1 * p=0 * p=1 \& p=2 \vee p=3 \circ p=4$

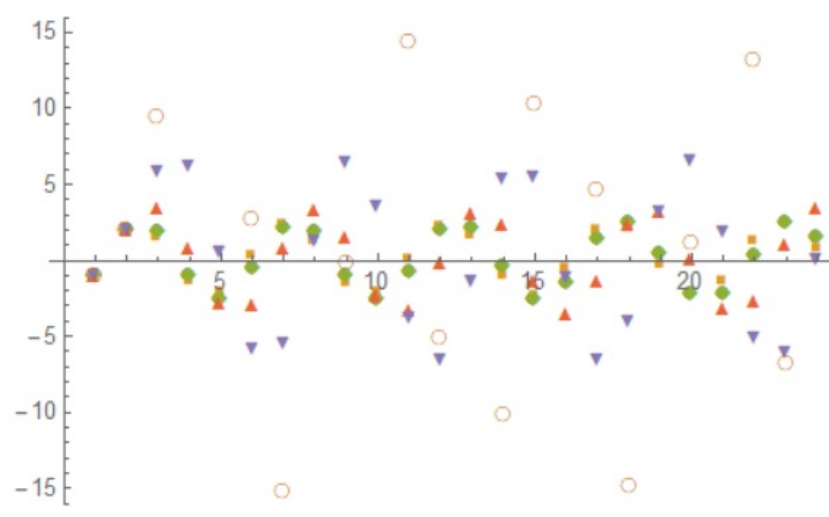

Figure 12: Comparison of varphi (n) for different $\mathrm{p}$ values, $\lambda=1, q=$ $\frac{1}{\sqrt{n}}$

- $\mathrm{q}=0 \quad \mathrm{q}=1 \quad \mathrm{q}=1 / \mathrm{n} \quad \triangle \mathrm{q}=1 / \sqrt{n}$

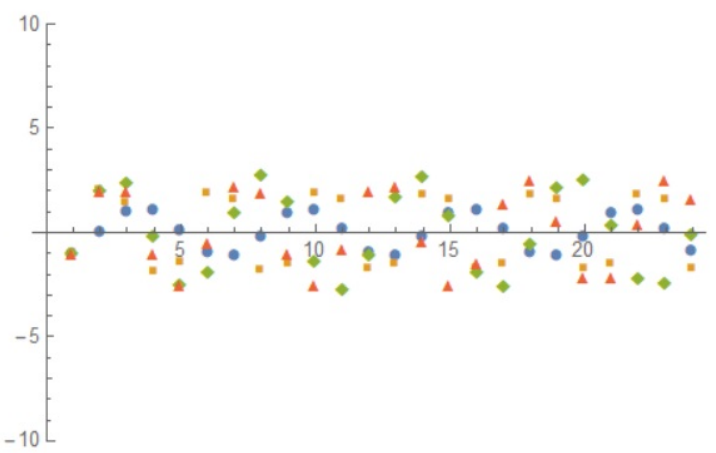

Figure 13: Comparison of $\varphi(n)$ for different potentials, $\lambda=1$

- $\lambda=1 \quad \lambda=2-\sqrt{3} * \lambda=2+\sqrt{3}$

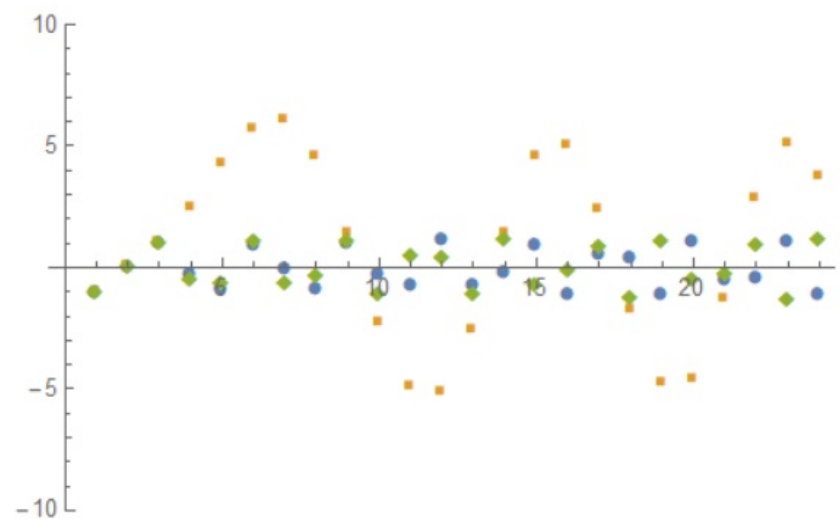

Figure 14: Comparison of $\psi(n)$ for different eigenvalues, $q=\frac{1}{\sqrt{n}}$
- $\mathrm{q}=0$ * $\mathrm{q}=1 * \mathrm{q}=1 / \mathrm{n} \quad \mathrm{q}=1 / \sqrt{n}$

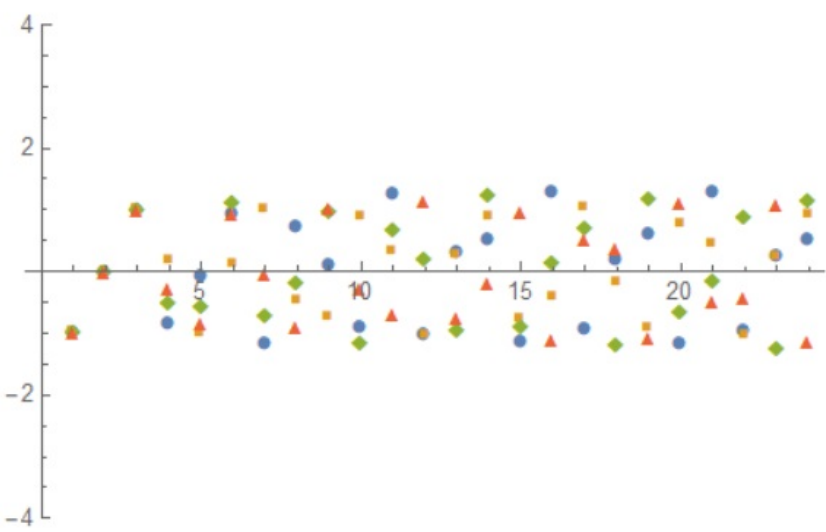

Figure 15: Comparison of $\psi(n)$ for different potentials $\lambda=2+\sqrt{3}, l=$

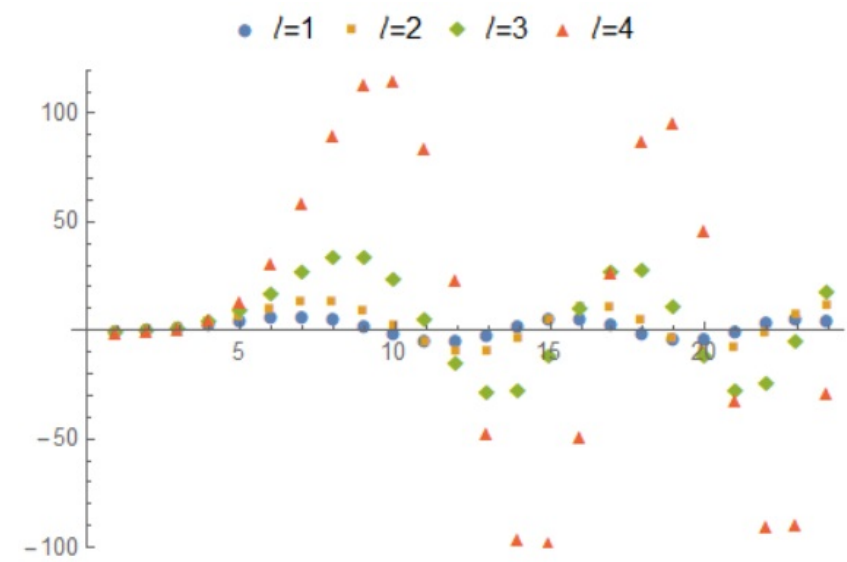

Figure 16: Comparison of $\psi(n)$ for different $l$ orbital quantum numbers, $\lambda=2+\sqrt{3}, q=\frac{1}{\sqrt{n}}$

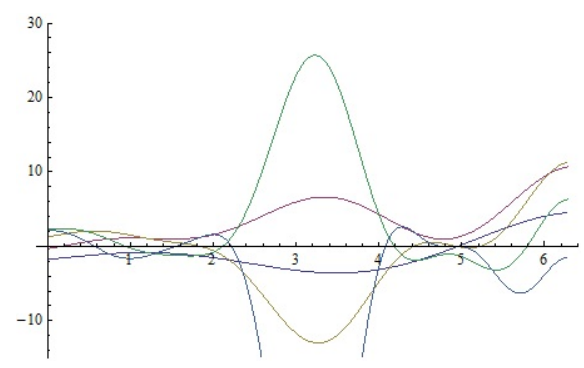

Figure 17: $\varphi(n), n=1,2, \ldots, 5$ 


\section{Conclusion}

In this study, we are concerned with discrete analogue of Sturm-Liouville problem with Bessel and hydrogen atom potentials (6) - (7) , (4) - (5), and we provide a basis for direct and inverse problems. From this point of view, we obtain representation of solutions, asymptotic estimations of eigenfunction and some numerical estimations about behaviors of eigenfunctions and eigenvalues. The numerical results for eigenfunctions corresponding to the certain significant eigenvalues for Sturm-Liouville problem having Bessel and hydrogen atom potential type are shown and compared to the each other. Our main aim is to apply the spectral theory of these type of differential equations to the discrete case.

\section{References}

[1] Boumenir A., Chanane B., Computing eigenvalues of SturmLiouville systems of Bessel type, Proc. Edinburgh Math. Soc., 1999, 42.2, 257-265.

[2] Bas E. Fundamental spectral theory of fractional singular SturmLiouville operator, J. Func. Spaces Appl., 2013.

[3] Levitan B.M., Sargsjan I.S., Introduction to Spectral Theory: Selfadjoint Ordinary Differential Operators, 1975, Amer. Math. Soc. 5-8.

[4] Epelde García, M., Bessel functions and equations of mathematical physics. Final Degree Dissertation, 2015, Universidad del País Vasco.

[5] Zakharov V., Bessel functions and their applications to solutions of partial differential equations, 2009, Math. 456 Lecture Notes.

[6] Albeverio S., Hryniv R., Mykytyuk Y., Inverse spectral problems for Bessel operators, J. Diff. Eq., 2007, 241(1),130-159.

[7] Everitt W.N., A catalogue of Sturm-Liouville differential equations. In Sturm-Liouville Theory, Birkhäuser Basel, 2005, 271331.

[8] Adkins C.J., Sturm-Liouville Theory, MSc thesis, 2014, University of Toronto.

[9] Abell M.L., Braselton J.P., Giving Meaning to the Circular Membrane Problem with Mathematica, Mathematica in Education, 1992, 1(3), 11.

[10] Bohner M., Cuchta T., The Bessel difference equa tion, Proc. Amer. Math. Soc., 2017, 145(4), 1567-1580.

[11] Panakhov E.S., Yilmazer R., A Hochstadt-Lieberman theorem for the hydrogen atom equation, Appl. Comp.Math., 2012, 11(1), 7480 .
[12] Bas E., Panakhov E., Ylmazer R., The Uniqueness Theorem for Hydrogen Atom Equation, TWMS J. Pure Appl. Math., 2013, 4, 20-28.

[13] Alghamdi A.M., Gala S., Ragusa M.A., A regularity criterion of weak solutions to the 3D Boussinesq equations, AIMS Math., 2017, 2(3), 451-457.

[14] Gala S., Ragusa M.A., On the regularity criterion of weak solutions for the 3D MHD equations, Z. Angew. Math. Phys., 2017, 68(6), 140.

[15] Moroşanu G., Second order difference equations of monotone type, Num. Func. Anal. Opt., 1979, 1.4, 441-450.

[16] Jirari A., Second Order Sturm-Liouville Difference Equations and Orthogonal Polynomials, Memoirs Amer. Math. Soc., 1995, 113, 542.

[17] Bender C.M., Orszag S.A., Advanced Mathematical Methods for Scientists and Engineers: Asymptotic Methods and Perturbation Theory, 1999, Springer-Verlag, Newyork.

[18] Agarwal R.P., Difference Equations and Inequalities, Marcel Dekker, 2000, 970, New York.

[19] Elaydi S., An introduction to difference equations, 2005, Springer Science+ Business Media, New York.

[20] Adivar M., Bairamov E. Spectral Properties of Non-selfadjoint Difference Operators, J. Math. Anal. Appl., 2001, 261, 461-478.

[21] Adivar M., Bairamov E., Difference equations of second order with spectral singularities, J. Math. Anal. Appl., 2003, 277, 714721.

[22] Akbulut A., Adivar M., Bairamov E., On the spectrum of the difference equations of second order, Publicationes Mathematicae-Debrecen, 2005, 67.3-4, 253-263.

[23] Aydemir K., Mukhtarov O.S., Asymptotics eigenvalues form many-interval Sturm-Liouville problems, AIP Conference Proceedings, AIP Publishing, 2016, 1726, 020088.

[24] Atkinson F.V., Discrete and Continuous Boundary Value Problems, 1964, Academic Press, New York.

[25] Kelley W.G., Peterson A.C., Difference Equations: An Introduction with Applications, 2001, Academic Press, San Diego.

[26] Bas E., Ozarslan R., Sturm-Liouville Problem via Coulomb Type in Difference Equations, Filomat, 2017, 31(4), 989-998.

[27] Bas E., Özarslan R., Spectral results of Sturm-Liouville difference equation with Dirichlet boundary conditions, In: Int. Conf. Adv. Nat. Appl. Sci., ICANAS 2016, AIP Publishing, 2016, 020065.

[28] Bas E., Ozarslan R., Asymptotics of eigenfunctions for Sturm- Liouville problem in difference equations, In: Int. Conf. Num. Anal. Appl. Math.: ICNAAM 2015, AIP Publishing, 2016, 290003.

[29] Panakhov E.S., Ercan A., Stability Problem of Singular SturmLiouville Equation, TWMS Journal of Pure and Applied Mathematics, 2017, 8(2), 148-159.

[30] Aydemir K., Mukhtarov 0.S., Asymptotics eigenvalues formanyinterval Sturm-Liouville problems, AIP Conf. Proc., AIP Publishing, 2016, 1726, 020088. 